\title{
Arbor
}

\section{Las tareas pendientes en política sanitaria}

\section{Pedro Sabando Súarez}

Arbor CLXXX, 710 (Febrero 2005), 431-440 pp.

Con la recuperación de la democracia, en el Sistema Sanitario español se han producido cambios importantes, especialmente significativos durante los años 80 y 90.

En estos años, España consolidó el Estado de Bienestar, pese a los problemas económicos tanto a nivel nacional como internacionales, siendo el Sistema Sanitario uno de sus pilares.

El punto de partida era ciertamente complicado. El Sistema Sanitario Público (S.S.P.), estaba configurado por múltiples redes asistenciales sin coordinación.

La estructura sanitaria a nivel ministerial dependía de 53 unidades. También había Diputaciones Provinciales y Ayuntamientos con responsabilidades sanitarias y estas administraciones eran titulares del $28 \%$ de las camas hospitalarias.

Nuestra Constitución de 1978 establece el derecho de los españoles a la protección de la salud, y por otro lado configura la estructura del Estado en Comunidades Autonómicas (CC.AA.)

Hagamos, pues, una muy somera revisión por periodos, para acercarnos a la problemática actual.

Entre los años 1975-1982 se mantiene el sistema heredado de la dictadura caracterizado por un significativo hospitalo-centrismo en un escenario de protesta e insatisfacción tanto de la ciudadanía como de los profesionales.

Entre los años 1982-1990, se desarrolla y extiende el sector público sanitario, definiéndose el Sistema Nacional de Salud (S.N.S.) en la Ley Feneral de Sanidad (L.G.S.) de 1986, entre cuyos grandes principios

' $\eta$ los siguientes: 
Desarrollo del S.N.S., a partir de los Servicios de Salud de las CC.AA., que integraban todas las redes públicas de asistencia sanitaria Cobertura universal y gratuita.

Financiación pública a expensas de impuestos.

Organización territorial de los servicios de salud en áreas y zonas básicas.

Pese a la L.G.S. y porque la propia ley lo hacía posible no se abordaron decisiones determinantes respecto a la financiación; la cobertura; la organización, incluído el ritmo del proceso de transferencias a las CC.AA., o la gestión de los distintos niveles del sistema.

A finales de los años 80 comienza a formularse como un problema determinante del futuro el crecimiento del gasto sanitario, que se perfila como un capítulo definitorio a la vez que de nuevo emerge la insatisfacción de ciudadanos y profesionales con el Sistema. El incremento de las listas de espera y su utilización adversarial en el ámbito político, es uno de los rasgos más tangibles, en un análisis superficial y de carácter inmediato que pone de manifiesto en forma aguda la falta de instrumentos y posibilidades económicas para que los gestores implementaran las reformas establecidas en la normativa vigente, utilizando incentivos en orden a reconocer el esfuerzo y dedicación de los profesionales.

Entre los años 1990 a 2000 la insatisfacción ciudadana crece, lo que conduce a múltiples iniciativas tanto dirigidas a conocer lo más científicamente posible, cual era la valoración del Sistema por los ciudadanos como a tomar o intentar tomar decisiones dirigidas a reformar la organización y la gestión del propio S.S.P.

En 1991, la llamada Comisión Abril presentó su informe que fue formalmente desestimado por el Gobierno, atendiendo a la contestación social en contra de políticas que podrían entenderse como fórmulas privatizadoras.

Pese a la declaración formal del Gobierno del momento, tanto en la red sanitaria dependiente del Gobierno Central como en algunos Sistemas de Salud Autonómicos, de todo signo político, se introdujeron diversas medidas señaladas como necesarias en dicho informe.

En 1996,otra Comisión, esta vez de carácter parlamentario, se desarrolla con la finalidad de analizar el sistema y ofrecer soluciones. El resultado, en modo alguno se acercó a la Comisión Abril ni analítica, ni propositivamente.

Tuvo sin embargo, a mi juicio, gran importancia política.

El hecho de que el Partido Popular (P.P.) entonces gobernando y con un previsible largo horizonte de gobernabilidad aceptara implícitamente 


\section{Las tareas pendientes en política sanitaria}

la L.G.S., muy criticada en su día desde el P.P. y sus ámbitos de influencia, otorgaba a dicha comisión especial relevancia.

La cobertura universal, la financiación pública, la descentralización a través de las correspondientes transferencias y el especial modo de vinculación de los profesionales al Sistema, quedaba ratificado, de tal suerte que, de hecho, en ese Diciembre de 1997 todo el arco parlamentario de España vendría a consolidar lo que había sido juzgado severamente, como atrevidas propuestas, once años antes en la L.G.S. por la oposición conservadora.

De alguna manera, podíamos hablar que en ese momento el S.S.P. quedaba consolidado políticamente y a salvo de incertidumbres por las alternancias en la gobernabilidad.

El futuro abría las puertas a los mecanismos de mejora en distintos aspectos; y se diluía la enmienda a la totalidad del Sistema.

Se trabajó en la contención de gastos, tanto en el sector farmacéutico y tecnológico como en otros capítulos, abordándose también el sistema de financiación autonómico que ofrecería una nueva formulación para el correspondiente capítulo sanitario.

Entre los años 2000 a 2004, continúan planteándose problemas estructurales del Sistema, que afectan a la racionalidad, a la gobernabilidad, a los ciudadanos como potenciales pacientes, a la situación de los hombres y las mujeres que dedican su vida al S.S.P., a la financiación sanitaria insuficiente, así como a la calidad y coordinación, viniendo a recoger y desarrollar lo que desde 1986 había quedado establecido como fundamento del Sistema.

En este periodo se promulgan la Ley de Cohesión y Calidad del S.N.S. y la Ley de Ordenación de las Profesiones Sanitarias. Ambas son normas apreciables; pero que no despejan la necesidad de una Ley General de Sanidad del Siglo XXI.

En octubre de 2004, el Presidente del Gobierno de España reúne la Conferencia de Presidentes Autonómicos, adquiriendo el Sector Sanitario el carácter de prioridad en la Agenda Política del Gobierno, de tal suerte que en la Conferencia de Presidentes, a celebrar en los próximos meses, el orden del día es monográficamente sanitario.

Hecha esta somera revisión del Sistema, y en espera de acontecimientos tan relevantes para el futuro del Sistema Sanitario como el que acabo de señalar, creo que es necesario decir que S.N.S., aunque no está en situación crítica, no disfruta de salud adecuada.

No es sencillo establecer un diagnóstico y sobre todo un pronóstico preciso porque sobre el actual S.N.S gravitan una serie de vectores críticos, a saber: 
a. El Sector Sanitario nunca había sido hasta ahora una prioridad en las Agendas Políticas, lo que ha determinado falta de poder y de recursos económicos propiciando un modelo retórico en la aplicación de las reformas. Esta realidad, junto a la necesidad política de evitar conflictos ha inhibido los compromisos de reforma, enunciándose los cambios como procesos intemporales, quedando buena parte de las propuestas en un marco declarativo.

b. La valoración política de la realidad sanitaria suele moverse entre la marcada complacencia de los Gobiernos y la mordacidad catastrofista de quien está en la oposición. Este modelo adversarial presta poca ayuda a buscar soluciones a los viejos y nuevos problemas.

c. Las asociaciones Profesionales y Sociedades Científicas, más allá de cualquier responsabilidad, imputan únicamente a los gobiernos todos los problemas. Podría decirse que los principales agentes del sistema sanitario público tienen una fuerte desafección respecto al mismo, que contrasta con la posición estratégica de la que gozan a la hora de colaborar en su gobierno y mejora.

d. Los Sindicatos no suelen contemplar el Sistema Sanitario Público como patrimonio de todos. Aunque hay intentos de asumir un protagonismo social, muchas veces las iniciativas de centrar una acción social responsable naufragan ante la tentación periférica de un sindicalismo reivindicativo, especialmente confortable en entornos públicos donde se goza de un plus de seguridad y cotas de influencia. Es indispensable que los agentes sociales sientan la sanidad pública como un patrimonio de todos.

e. Una vez producidas las transferencias a las Comunidades Autó nomas los desequilibrios amenazan al sistema. Los errores económicos en los traspasos han sido importantes y el sistema de financiación no invita al optimismo. La complacencia de los ministros económicos del gobierno central que hizo las últimas transferencias en Diciembre 2001, con el nuevo modelo de financiación autonómica y el pacto de estabilidad presupuestaria, también provenía de la inconfesable alegría por haber expulsado un área inflacionaria y de propulsión de déficit de su ámbito de gestión, para pasar la "patata caliente» a las administraciones autonómicas. Sin embargo el problema sigue planteado en términos de compromiso e implicación de todos los poderes públicos, y el papel de la Administración Central del Estado y su responsabilidad en la tutela financiera del sistema sanitario es imposible de obviar. 
Las tareas pendientes en política sanitaria

f. El Gasto sanitario crece aceleradamente en relación con el PIB, lo que haría necesario en esta etapa un proceso de «normalización» con la media del gasto en los países de nuestro entorno de la Unión Europea.

El aumento y envejecimiento de la población, la especifica repercusión en el S.S.P. de la inmigración, el aumento de las expectativas de salud de los ciudadanos y la medicalización de la sociedad configuran un escenario que supone un notorio incremento de la demanda de servicios sanitarios.

Paralelamente la utilización en los diagnósticos y tratamientos de nuevas tecnologías junto al encarecimiento de las terapias farmacológicas y del gasto farmacéutico en su conjunto establecen un marco que explica las causas más relevantes del incremento en el gasto señalado.

g. Los medios de comunicación contemplan el Sistema Sanitario en términos manifiestamente mejorables. Desfiguran de tal manera la realidad que la hacen incomprensible para el ciudadano atento.

Ante este escenario, tendrá lugar la Conferencia de Presidentes Autonómicos bajo la presidencia del Sr. Rodríguez Zapatero, y la financiación de la sanidad será una de sus principales ocupaciones.

No puedo por tanto, en estos días, escribir un articulo sobre nuestra Sanidad, sin hacer algunas consideraciones acerca de la financiación sanitaria.

En las transferencias de los recursos sanitarios del INSALUD, a las CC.AA de la llamada "vía lenta", producida el 31 diciembre del 2001, se negociaron inadecuadamente algunos capítulos, dos ejemplos:

Se aceptaron cifras globales inferiores a las de la liquidación del INSALUD de dos años antes, esto es de 31/12/1999, asumiendo por tanto un escenario de subfinanciación.

No se contempló la población real en ese momento al aceptar como adecuada la de 1999 habiéndose producido un incremento significativo en esos dos años, que en Madrid fue en torno a 800.000 personas.

Errores de esta naturaleza han contribuido decisivamente a situar a algunos Sistemas de Salud de distintas CC.AA en una situación de subfinanciación crónica que se deberá abordar especifica, pormenorizada y diferenciadamente, pues no debe confundirse con ciertos déficit del que verosímilmente deberán responder las correspondientes Comunidades Autónomas.

En todo caso los recursos financieros que se transfieran a las CC.AA, deberán tener carácter finalista sanitario. 
Varios pueden ser los escenarios de trabajo en orden a la financiación sanitaria.

Teniendo en cuenta que todos los grupos parlamentarios en todas las instituciones de España expresan respeto y admiración por nuestro sistema y sus profesionales sería bien lógico que se produjera un Pacto de Estado por la Sanidad que asegurara la sostenibilidad del sistema adoptando las formas que se consideraran adecuadas teniendo en cuenta los precedentes habidos en dichas materias.

También se podría avanzar estableciendo mecanismos para solucionar los problemas puntuales de cada Sistema Regional de Salud en espera de un abordaje global.

La posibilidad de incrementar expresamente para la financiación sanitaria la presión fiscal de una manera específica no debería descartarse.

En los medios de comunicación está también la posibilidad de que las CC.AA pudieran mejorar la recaudación, o bien aplicar impuestos sobre el alcohol y el tabaco que deberían tener también el carácter de finalistas.

Por supuesto, tal como señalaba el Presidente de la Federación de Asociaciones para la Defensa de la Sanidad Pública en su reciente comparecencia en la Asamblea de Madrid los Fondos de Cohesión deben ser modificados. Estima el Doctor Sánchez Bayle que precisarían un incremento económico del 1\% del gasto sanitario publico.

En cualquier caso, hay que felicitarse porque esta materia será tratada en la Conferencia de Presidentes y a todos nos corresponde desarrollar una auténtica tormenta de ideas porque la trascendencia es máxima. Detrás de ella está la pervivencia y el desarrollo de un Sistema que cuida de la salud de los ciudadanos, crea riqueza económica e intelectual y forma profesionales de nivel adecuado que cumplen sus funciones tanto en el orden asistencial, como en el docente e investigador.

Corresponde a la izquierda señalar los riesgos de crisis, para evitarla, porque si hubiera que resolver un trance agudo y urgente suele apelarse a tratamientos de emergencia que tienden a afectar a la parte más débil del sistema social, como se ha demostrado en los acontecimientos de la sanidad alemana.

La propuesta del copago emerge de nuevo bajo distintas fórmulas, con respecto a la visita médica, tanto en Alemania, como en Francia o en Italia. Delicada hipótesis de trabajo; intensa y pormenorizadamente debatida sin que llegara a considerarse un procedimiento adecuado ni en la perspectiva disuasoria ni en la financiera; pero sin duda el futuro obligará a la búsqueda de una fórmula que conduzca a una mayor responsabilidad individual en la utilización del Sistema Sanitario. 
Buena parte de los problemas que hoy padecemos, provienen de la dificultad creciente de mantener sectores de bienestar social (y en particular el de la sanidad pública) mayoritariamente fuera de la influencia del mercado, en un contexto social donde la lógica del capital ha ido avanzando, y donde la ética más insolidaria del beneficio privado sin responsabilidad social parece haberse extendido. Es difícil evitar que esta lógica y esta ética de mercado se filtren por todos los poros de los servicios públicos, y afecten negativamente a su compromiso y moral de trabajo. Los problemas se agravan cuando, no se asume con convicción la necesidad de preservar de la lógica del mercado a los servicios sanitarios y responder con la ética pública y la democracia sanitaria a los retos que hoy enfrentamos.

Preservar el S.N.S., es una exigencia de nuestra sociedad y una garantía de cohesión social, por ello, ante las incertidumbres económicas y la persistencia de problemas estructurales, llama la atención el discurso complaciente, cuando el grado de vulnerabilidad del sistema se ha incrementado tras el proceso transferencial.

A la izquierda le corresponde, también, plantear fórmulas a desarrollar, llevarlas a cabo donde gobernamos, y hacer un seguimiento inteligente y pormenorizado desde la oposición, cuando corresponda.

Estamos pues, ante una situación compleja y por su propia naturaleza mal definida.

A través de cinco capítulos se pueden marcar las líneas maestras de orientación dirigidas a evitar la temida crisis y que integran conceptos esenciales:

\section{Primero:}

Es necesario, cuando menos, mantener actualizadas las prestaciones del S.N.S.; sobre ello pueden incidir los cambios poblacionales y tecnológicos, generando desequilibrios que no deben recaer sólo en las Comunidades Autónomas, sino que deberán ser problemas de Estado, a solucionar a través del Consejo Interterritorial.

\section{Segundo:}

Hay que defender la equidad y la calidad del Sistema para evitar la deslegitimación y la privatización inducida por deterioro de los servicios públicos. Evitemos la miopía: el principal riesgo de privatización hoy no 
está encima de la mesa, sino que subyace a la propia incapacidad de los servicios públicos para dar respuestas apropiadas a los ciudadanos, a sus necesidades, demandas y preferencias; lo grave de la situación es que la mera falta de acción y por supuesto, las posiciones defensivas e insolidarias llevan a una pérdida creciente de funcionalidad, reputación y credibilidad.

La equidad se resiente gravemente cuando los ciudadanos tienen distintas posibilidades de obtener servicios públicos sanitarios en función de características personales (económicas, culturales y sociales) El Sistema Nacional de Salud que la izquierda quiere para España ha de ser un servicio público de calidad al que todos debemos contribuir según nuestra renta, y del que todos podemos beneficiarnos según nuestra necesidad.

\section{Tercero:}

El sistema ha de gestionar eficientemente los cambios científicos y tecnológicos discriminando la innovación útil en orden a optimizarla en la mejora de salud de los ciudadanos.

Este capítulo supone la reflexión en torno a la sostenibilidad del sistema, sobre la que gravitan factores ajenos al mismo (sostenibilidad externa), y otros que cristalizan en los propios agentes del sistema (sostenibilidad interna).

La sostenibilidad externa depende fundamentalmente de cómo se gestiona la innovación en medicamentos y en electromedicina. El gasto público en medicamentos crece a un ritmo insostenible a corto plazo; pero lo grave es que el incremento anual, en torno a un $10 \%$, no se corresponde con un incremento similar en la mejora de problemas de salud sino que muchas veces sólo se corresponde con incrementos marginales de eficacia justificados a través de estudios donde con frecuencia se bordean los límites de la metodología científica, para magnificar efectos y utilidades de es casa entidad, y poner sordina a los inconvenientes y efectos adversos. La menguada eficacia de la innovación se compensa con amplias campañas de marketing que con frecuencia pervierten la lógica razonable y cooperativa de relación entre profesionales, sistema e industria, generando conflictos de interés que afectan a la motivación de los profesionales.

El sector industrial en algunos casos quiere mantener beneficios que no se corresponden con la eficiencia social añadida que aportan. Los Sistemas Sanitarios pueden y deben defender el buen uso de sus recursos a través de procesos de evaluación de efectividad y eficiencia social de medicamentos y tecnologías, así como desarrollar las correspondientes polí- 
ticas de uso racional. La industria farmacéutica y electromédica tienen un papel esencial en la creación de valor y en el apoyo a buscar nuevas alternativas diagnósticas y terapéuticas; pero deben de recibir señales adecuadas por parte de las autoridades sanitarias, científicas y profesionales, para que su esfuerzo se dirija a la innovación que realmente aporte valor a los pacientes y a la sociedad.

La sostenibilidad interna depende de componentes propios o inherentes al sistema, como son las políticas dirigidas al factor humano que han de estar orientadas a mejorar la productividad social.

En esencia se trataría de hacer un tránsito fundamental: consolidar en una primera fase el cambio desde «políticas de personal» a "políticas de recursos humanos» (es decir reconocer el papel central de las personas y sus motivaciones en las organizaciones sanitarias y desarrollar mecanismos que garanticen el alineamiento de intereses entre los trabajadores y la organización); Pero en una segunda fase (y en ella debemos entrar lo antes posible), deberíamos complementar las políticas de recursos humanos con "políticas profesionales». Estas políticas incorporan nuevos elementos que parten de reconocer que los profesionales sanitarios son decisores y micro-asignadores de recursos, y que deben asumir dicha función a través de instrumentos de gestión del conocimiento, autonomía y corresponsabilidad, gestión clínica, y asunción de un nuevo papel de colaboración con la sostenibilidad general del sistema.

Esto es sin duda difícil; porque exige realinear los intereses de todos los agentes, y sobre esta base hacer un depósito de la confianza mutua, para dar un protagonismo fundamental a la ética de servicio a los pacientes en la resolución de problemas y conflictos.

\section{Cuarto:}

El S.N.S, ha de fijar unos objetivos que al ser comunes permitan establecer una dirección estratégica, desde el vértice institucional, que vertebre y conecte un sistema sanitario descentralizado.

El S.N.S. ha de desarrollar instrumentos horizontales, que permitan la gestión eficiente de procesos críticos como la función de compra y aprovisionamiento o los sistemas de evaluación y control.

La consolidación del S.N.S. pasa por dotar de una alta legitimidad social a los servicios sanitarios públicos, que también corresponde al vértice institucional del sistema y a las políticas que emanen del mismo.

No es fácil en sistemas tan descentralizados como el SNS encontrar modelos de gobernabilidad corporativa que puedan dar respuesta a los 
retos que enfrentamos; pero las decisiones están en todo caso muy lejos de reinventar papeles centrales de tipo jerárquico o planificador, y más cercanas a crear una estructura colaborativa de gestión del conocimiento y la innovación, de instrumentos colectivos de apoyo a la gestión y logística común del sistema. Creo que éste debe ser el modelo hacia el que debe orientarse el SNS, para ser dirigido a través del Consejo Inter-territorial. Posiblemente haga falta explorar nuevas ideas; ¿porqué no generar «de abajo a arriba» un Comisionado del SNS que gestione las competencias técnicas comunes?.

\section{Quinto:}

Es necesario crear plataformas de conocimiento relevante y compartido, financiando la difusión del mismo, promoviendo sinergias entre administraciones sanitarias para responder ordenadamente a los problemas de salud o de servicios sanitarios.

La gobernabilidad clínica, debe utilizarse como instrumento esencial de gestión del conocimiento científico para evaluar eficazmente la innovación relevante, y realinear los valores esenciales del profesionalismo sanitario con los de la sociedad. Entendido como una construcción común en la que debe participar lo mejor de las profesiones sanitarias, buscando una nueva alianza que permita reconstruir un ejercicio profesional digno, responsable, socialmente considerado, alejado de presiones y conflictos de interés, donde se reconozca el mérito, y se incentive la excelencia y la capacidad de responder de forma innovadora a necesidades y demandas de los pacientes

Todo ello, ha de promoverse desde el Consejo Interterritorial, ámbito específico y privilegiado de actuación que ha de ser la pieza institucional básica para el desarrollo de un S.N.S., tal como se formuló en la Ley General de Sanidad 14/86, que garantiza el derecho de todos los ciudadanos a un acceso equitativo a los servicios sanitarios por ser un sistema universal financiado públicamente.

Estas son las tareas pendientes en la sanidad, que exigen combinar solidaridad, innovación organizativa e inteligencia. Y en estas coordenadas tenemos la responsabilidad de alimentar el debate y la reflexión de futuro. 


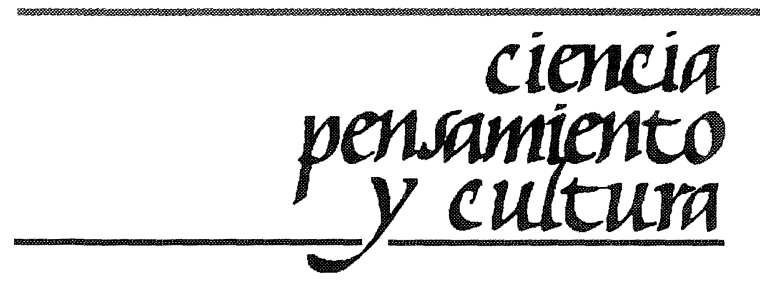

De próxima publicación:

ESPAÑOLES EN ORIENTE PRÓXIMO.

AVENTUREROS Y PEREGRINOS, MILITARES, CIENTÍFICOS Y

DIPLOMÁTICOS OLVIDADOS EN

EL REDESCUBRIMIENTO DE

UN MUNDO (1166-1923)

Joaquín M. ${ }^{a}$ Córdoba (Editor)

La Revista Arbor está incluída en el apartado de Arte y Humanides del CITATION INDEX

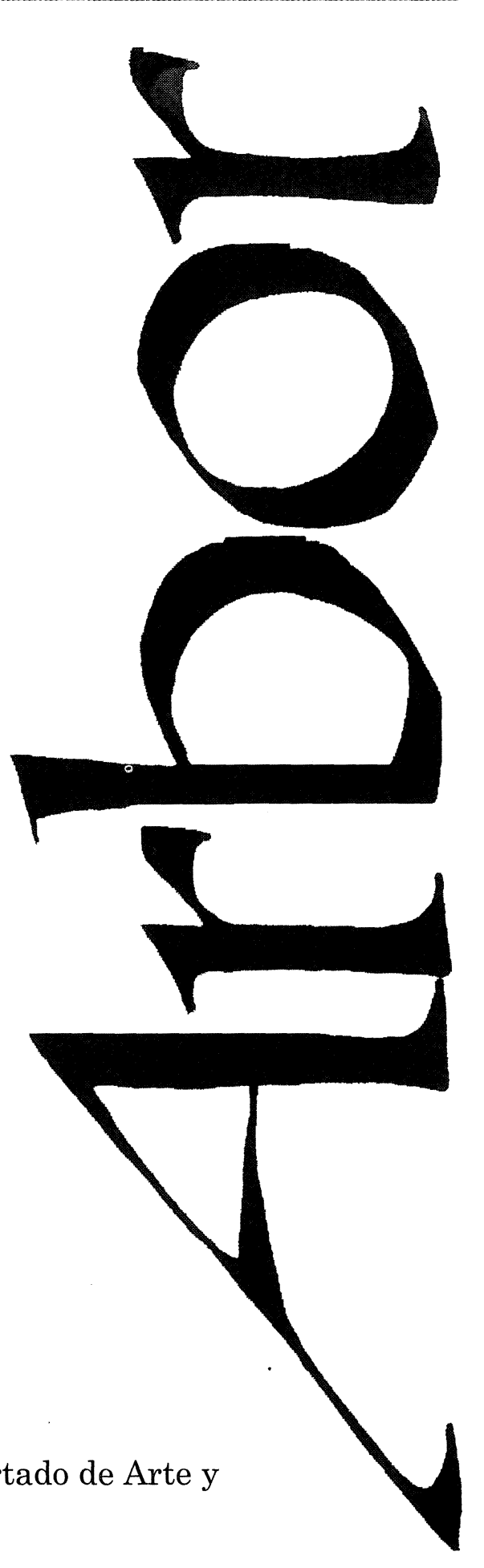

